\title{
Magnetic resonance imaging of the wrist in early rheumatoid arthritis reveals a high prevalence of erosions at four months after symptom onset
}

Fiona M McQueen, Neal Stewart, Jeff Crabbe, Elizabeth Robinson, Sue Yeoman, Paul L J Tan, Lachy McLean

\begin{abstract}
Objectives-To evaluate the role of magnetic resonance imaging (MRI) of the wrist in detecting early joint damage in patients with rheumatoid arthritis (RA). Methods-MRI was performed on 42 patients with early RA (median symptom duration of four months). Scans were scored separately by two musculoskeletal radiologists using a newly devised scoring system, which was validated. MRI findings were compared with plain radiography, clinical measures, and HLA-DRB ${ }^{\star} 01 / 04$ genotyping.

Results-Interobserver reliability for the overall MRI score was high $(r=0.81)$ as was intraobserver reliability $(r=0.94$ for observer 1 and 0.81 for observer 2 ). There was more variation in scoring synovitis (interobserver reliability: $r=0.74)$. Erosions were detected in $45 \%$ of scans (19 of 42 ), compared with $15 \%$ of plain radiographs. The most common site for erosions was the capitate $(39 \%)$, for synovitis the ulnar aspect of the radiocarpal joint, and for tendonitis, the extensor carpi ulnaris tendon. The total MRI score and MRI synovitis score correlated most significantly with $C$ reactive protein $(r=0.40$ and 0.42 respectively, $p<0.01$ ). The MRI erosion score was highly correlated with MRI bone marrow oedema $(r=0.83)$ as well as the Ritchie score and disease activity score $(r=0.32, p<0.05)$. HLA-DRB $1{ }^{\star} 04$ or $\star_{01}$ (shared epitope +ve) was found in $76 \%$ of patients; $84 \%$ of those with MRI erosions and $69 \%$ of those without (NS, p = 0.3).
\end{abstract}

Conclusions-A high proportion of RA patients develop MRI erosions very early in their disease, when plain radiography is frequently normal. MRI of the dominant wrist may identify those requiring early aggressive treatment.

(Ann Rheum Dis 1998;57:350-356)

Department of Molecular Medicine, Auckland School of Medicine, Auckland University, Private Bag 92019, Auckland, New Zealand

Correspondence to: Dr F McQueen.

Accepted for publication 29 April 1998
The natural history of rheumatoid arthritis (RA) during the first year after symptom onset remains poorly understood. Within this period, joint synovial inflammation progresses to erosion of cartilage and bone in up to $47 \%$ of patients, ${ }^{1}$ many of whom go on to develop severe, disabling disease. ${ }^{2}$ Plain radiography is normal in the majority at diagnosis ${ }^{3}$ and does not allow detection of early synovial inflammation, which may herald joint damage. Aggres- sive early intervention using immunomodulatory agents is therefore often delayed until the point when bony erosions are apparent radiologically and irreversible joint damage has already occurred.

Prognostic factors in early RA have recently been the subject of intensive investigation. ${ }^{4}$ Possession of one or two HLA-DRB1 alleles containing the "shared epitope" sequence (QK/RRAA at amino acid positions 70-74 of the HLA-DR $\beta$ chain $)^{5}$ has been associated, in a dose dependent fashion, with more severe disease in white populations. ${ }^{6}$ Other adverse prognostic factors include positivity for rheumatoid factor, clinical/laboratory measures of disease activity, low socioeconomic status ${ }^{7}$ and radiological abnormalities such as the presence of erosions. ${ }^{4}$ Erosions have been used as a poor prognostic sign in early RA and are also a traditional end point to evaluate disease progression. ${ }^{8}$ Ideally the prediction of poor outcome should be made before erosions develop so that treatment may be modified to try to prevent this. ${ }^{9}$ Clearly, there is a need to identify other parameters that can be evaluated in early RA to better predict outcome.

Magnetic resonance imaging (MRI) of the wrist has been shown in cross sectional analyses to be much more sensitive for the detection of early erosions in RA than plain radiography. ${ }^{10}{ }^{11}$ Moreover, MRI can reveal inflammatory changes within the soft tissues of the joint that are not visible on radiographs and may precede the development of erosions. Thus, carpal MRI could provide evidence for early aggressive RA and be of use in predicting long term prognosis. A scoring system applicable to MRI in this context must be developed to quantitate pathology within the joint, analogous to the radiological scoring systems of Sharp and Larsen..$^{12}$ The reliability of such a score needs to be assessed and performance compared longitudinally with conventional radiography for monitoring disease progression. We have devised such a system for scoring MRI of the wrist and report baseline findings for 42 patients with early RA in conjunction with clinical, laboratory, and HLA-DRB1 typing data. Prospective follow up of these patients will allow assessment of the clinical utility of MRI in early RA.

\section{Methods}

PATIENT POPULATION

Patients were referred to an early arthritis clinic by primary care physicians and specialist rheumatologists from hospital rheumatology 
Table 1 Patient characteristics at baseline $(n=42)$

\begin{tabular}{ll}
\hline Clinical features & \\
Age (y) median (range) & $48: 5(27-75)$ \\
Sex F:M & $25: 17$ \\
Ethnic group & 33 white, 5 Maori, 4 Pacific Island \\
Duration of symptoms (months) mean (range) & $4(2-6)$ \\
Seropositive (ever) & $38(90 \%)$ \\
Medications & \\
NSAID & $38(90 \%)$ \\
DMARD & $21(50 \%)$ \\
prednisone (5-13 mg) & $5(12 \%)$ \\
Disease activity & Median (range) \\
Ritchie index & $13.5(0-37)$ \\
Swollen joint count & $14.5(0-38)$ \\
Pain score & $3.3(0.7-9.3)$ \\
HAQ score & $0.6(0-1.8)$ \\
DAS & $4.19(1.25-7.57)$ \\
ESR (mm 1st h) & $29(10-131)$ \\
CRP (mg/l) & $18(0-150)$ \\
\end{tabular}

$\star$ See text.

outpatient clinics and private practice within the Auckland and Waikato areas of New Zealand after a media campaign. Approval for the study was granted by the North Health and Waikato Ethics Committees. Enrolment for the study proceeded from 1994-1996. Patients were recruited consecutively providing they met the 1987 revised American College of Rheumatology (ACR) criteria for rheumatoid arthritis ${ }^{14}$ and had symptoms of six months or less duration. MRI of the wrist at baseline and at one year were offered to 55 patients of whom 44 consented to be enrolled. Two of these patients were excluded because they exceeded the maximum weight for the scanner $(110 \mathrm{~kg})$, making a total of 42 patients. Table 1 gives patient characteristics.

\section{CLINICAL ASSESSMENTS}

Patients received a full clinical assessment by a rheumatologist at entry to the study including documentation of history, medications, scoring for the Ritchie index, ${ }^{15}$ swollen joint count (maximum possible 58), tender joint count (maximum possible 60 ), HAQ score ${ }^{16}$ visual analogue pain score, patient and physician global assessments, Steinbrocker functional class, ${ }^{17}$ disease activity score, ${ }^{18}$ and identification of extra-articular features. Standard laboratory tests included full blood count, erythrocyte sedimentation rate, $\mathrm{C}$ reactive protein (CRP), rheumatoid factor, and anti-nuclear antibodies. Clinical assessments are being repeated at six monthly intervals for a two year period but baseline data only are presented here. Fifty per cent (21 of 42) of patients were taking disease modifying anti-rheumatic drugs (DMARDs) at baseline, the majority receiving sulphasalazine (18 of 21) with two patients taking hydroxychloroquine and one methotrexate. However, most patients had only been taking DMARDs at full dose for less than one month (median duration, one week). Five patients $(12 \%)$ were taking prednisone at 5-13 $\mathrm{mg} / \mathrm{d}$ (median duration, five weeks) (table 1).

RADIOGRAPHY

Radiographs of the hands and feet using standard views were taken at baseline and are being repeated at one and two years. In each patient, films of the dominant carpus have been scored for erosions within the same field of view as
Table 2 MRI scoring system

\begin{tabular}{llll}
\hline MRI feature & $\begin{array}{l}\text { Possible } \\
\text { scores }\end{array}$ & $\begin{array}{l}\text { Number of } \\
\text { sites }\end{array}$ & $\begin{array}{l}\text { Maximum } \\
\text { score }\end{array}$ \\
\hline $\begin{array}{l}\text { Erosions } \\
\text { Bone oedema }\end{array}$ & $0,1,2$ & 15 & 30 \\
$\begin{array}{l}\text { Synovitis } \\
\text { thickening }\end{array}$ & $0,1,2$ & 15 & 30 \\
$\quad$ enhancement & $0,1,2$ & 7 & 14 \\
$\begin{array}{l}\text { Tendonitis } \\
\quad \text { enhancement }\end{array}$ & $0,1,2$ & 7 & 14 \\
$\quad$ size & 0,1 & 9 & 9 \\
$\quad$ sheath & $0,1,2$ & 9 & 18 \\
Total possible score & 0,1 & 9 & 9 \\
\hline
\end{tabular}

${ }^{\star}$ As detailed in the text.

Table 3 Validation of MRI scoring system

\begin{tabular}{lll}
\hline MRI score & Reliability & $95 \% C I$ \\
\hline Interobserver reliability & & \\
Synovitis & 0.74 & $(0.56,0.85)$ \\
Tendonitis & 0.77 & $(0.60,0.87)$ \\
Bone marrow oedema & 0.84 & $(0.72,0.91)$ \\
Erosion score & 0.77 & $(0.60,0.87)$ \\
Erosion number & 0.79 & $(0.64,0.98)$ \\
Total MRI score & 0.81 & $(0.64,0.89)$ \\
Intraobserver reliability & & \\
Observer 1 & & \\
Total score & 0.94 & $(0.66,0.99)$ \\
Erosion score & 0.98 & $(0.93,0.99)$ \\
Observer 2 & & \\
Total score & 0.81 & $(0.05,0.95)$ \\
Erosion score & 0.92 & $(0.48,0.98)$ \\
\hline
\end{tabular}

imaged on MRI scans, by two musculoskeletal radiologists using criteria defined by Sharp. ${ }^{12}$

\section{GENETIC STUDIES}

DNA was extracted from anticoagulated blood obtained from each patient at recruitment. Low resolution HLA-DRB1 typing was performed using sequence specific primer polymerase chain reaction (SSP-PCR) with a standardised panel of 24 oligonucleotide primer pairs. ${ }^{19}$ In subjects with alleles of the $\mathrm{DRB} 1^{\star} 04$ or 01 groups, the sequence of the subtype determining region of exon 2 of the DRB1 gene(s) was obtained by direct sequencing of PCR products, ${ }^{20}$ to be reported in detail separately. Allele specific primers ${ }^{21}$ were modified by the addition of standard M13 sequences to their 5' tails. PCR products were purified using High Pure columns (Boehringer Mannheim) then sequenced using standard dye labelled M13 primers and AmpliTaq FS DNA polymerase (Perkin Elmer) on an ABI373 automated fluorescent sequencer (ABI Prism).

MRI

MRI of the dominant wrist was undertaken using a 1.5 Tesla MR scanner (GE Signa Horizon) with a dedicated wrist coil (Medical Devices). The field of view was $8 \mathrm{~cm}$ and included the distal radioulnar, radiocarpal, and midcarpal joints as well as the metacarpal bases. The small field of view was chosen to optimise resolution. The metacarpophalangeal (MCP) joints were therefore not included. The imaging protocol comprised firstly, coronal and axial T1 sequences, followed by axial fat suppressed fast spin echo T2, then coronal fat suppressed $\mathrm{T} 1$ sequences after injection of gadolinium (Nicomed Omniscan). A dynamic series was performed and will be the subject of a separate report. Static scans were scored 

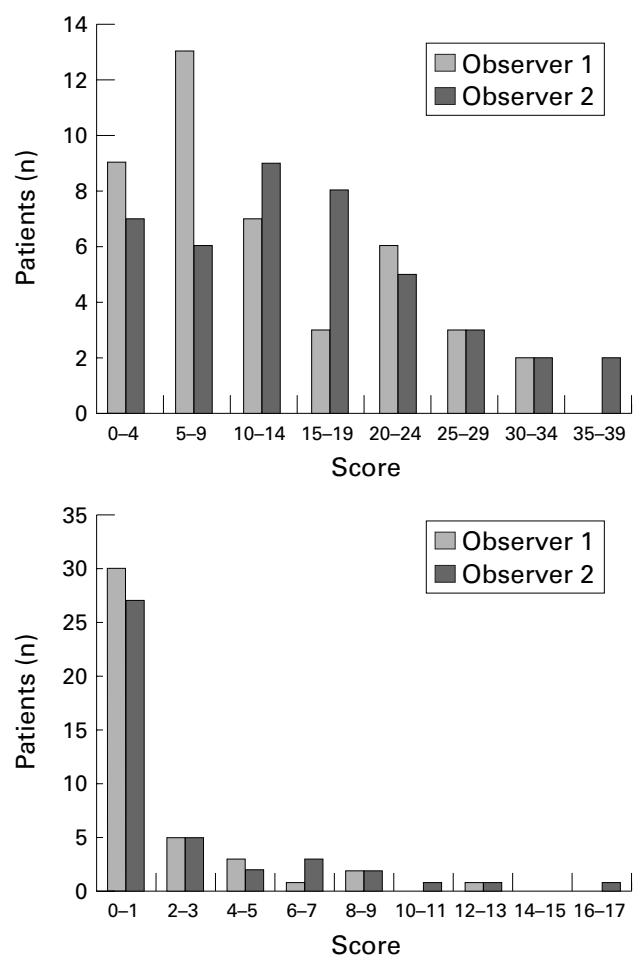

Figure 1 MRI erosion score (lower panel) and total score (upper panel) for observer 1 and observer 2.

Table 4 Details of findings on MRI $(n=42)$

\begin{tabular}{lll}
\hline MRI feature & $\% M R I^{\star}$ & Most common sites (\%)* \\
\hline Erosions & $55(45 \dagger)$ & $\begin{array}{l}\text { capitate (39) } \\
\text { lunate (29) } \\
\text { triquetrum (26) } \\
\text { lunate (33) } \\
\text { triquetrum (24) } \\
\text { capitate (22) } \\
\text { radiocarpal - ulnar (88) } \\
\text { Badiocarpal - radial (88) } \\
\text { intercarpal (81) } \\
\text { oedema }\end{array}$ \\
Synovitis & 64 & $\begin{array}{l}\text { flensor carpi ulnaris (33) } \\
\text { extensor carpi radialis }(26)\end{array}$ \\
Tendonitis & 79 & \\
\end{tabular}

$\star \%$ of MRI scans where abnormality identified by one or both observers. †Consensus opinion for observers 1 and 2 .

independently by two musculoskeletal radiologists for bone oedema and erosions, synovitis, and tendonitis after maximising concordance using three scans outside the study. The radiologists were blinded to plain radiograph results when scoring MRI. We present baseline MRI and radiological data in this report. Imaging is being repeated at one year and these comparative data will be the subject of a future report.

MRI SCORING SYSTEM

Erosions were identified by focal loss of normal low signal intensity from cortical bone on $\mathrm{T} 1$ weighted images, increased signal on T2 weighted images and enhancement with gadolinium implying the presence of inflamed synovium within the defect. Each of the 10 carpal bones and the bases of the five metacarpal bones were assessed for erosions (total of 15). Erosions were scored on size as $0,1(<4$ $\mathrm{mm}$ diameter) or 2 ( $>$ than $4 \mathrm{~mm}$ in diameter). Erosions at each site were counted (erosion number) and an erosion score per site was obtained. Scores were added to give totals for the carpus (maximum possible erosion score $=$ 30) (table 2). Bone marrow oedema was scored at the same sites ( 0 for none, 1 for minor oedema involving $<50 \%$ of the bone, 2 for gross oedema involving $>50 \%$ of the bone marrow). The total bone marrow oedema score was obtained from the sum of all scores (maximum possible $=30$ ).

Synovitis was assessed at seven individual sites within the carpus; the distal radioulnar joint, radiocarpal joint (ulnar aspect), radiocarpal joint (radial aspect), intercarpal joint (between the proximal and the distal carpal row), carpometacarpal joints (2-5 assessed together), 1st carpometcarpal joint, and the pisotriquetral joint. These have been used in a previous MRI scoring system described by Ostergaard et $a l^{22}$ with the addition of the pisotriquetral joint, which could be seen on our axial scans. Synovitis was scored using synovial thickening ( 0 for $<2 \mathrm{~mm}, 1$ for $2-4 \mathrm{~mm}$ and 2 for $>4 \mathrm{~mm})$ and post-gadolinium enhancement ( 0 for none, 1 for mild to moderate and 2 for gross). Scores were added to give a total for the carpus (maximum possible $=28$ ) (table 2$)$.

Nine tendon groups were scored including extensor pollicis brevis, extensor carpi radialis, extensor pollicis longus, extensor digitorum, extensor digiti minimi, extensor carpi ulnaris, flexor carpi radialis, flexor digitorum longus, and flexor carpi ulnaris. They were evaluated on the axial scans using $\mathrm{T} 1$ and $\mathrm{T} 2$ sequences. A score of 0 (if absent) or 1 (if present) was given for inflammation in the tendon sheath (increased bright signal around the tendon on T2W images and $>1 \mathrm{~mm}$ thickness of sheath on two consecutive slices). Inflammation within the tendon itself was also scored $(0=$ normal signal, $1=$ increased signal on $\mathrm{T} 1$ and/or $\mathrm{T} 2$ sequences). Tendon size was scored as 0 for normal, 1 for thickened or 2 for attenuated. Scores were added for a total tendon/sheath score for the carpus (maximum possible $=36$ ). A total MRI score for the carpus was derived from total scores for erosions, bone marrow oedema, synovitis, and tendonitis (maximum possible $=124)($ table 2$)$.

\section{STATISTICS}

Intraclass correlation coefficients were calculated to investigate the inter-rater and intrarater reliability for MRI scores. ${ }^{23}$ This method was not appropriate for use with the data from plain radiographs as this followed a nonparametric distribution. Percentage agreement for the presence/absence of erosions between and within observers is therefore presented. The Wilcoxon signed rank test was used to investigate differences in MRI scores between the two raters. Differences in patient characteristics between the MRI group and others in the trial were analysed using $t$ tests, Mann-Whitney $\mathrm{U}$ or $\chi^{2}$ tests as appropriate. Pearson's correlation coefficients were calculated between clinical and MRI scores for individual raters and then combined using Fisher's transformation. ${ }^{24}$ Fisher's exact test was used to examine differences in proportions between those with 

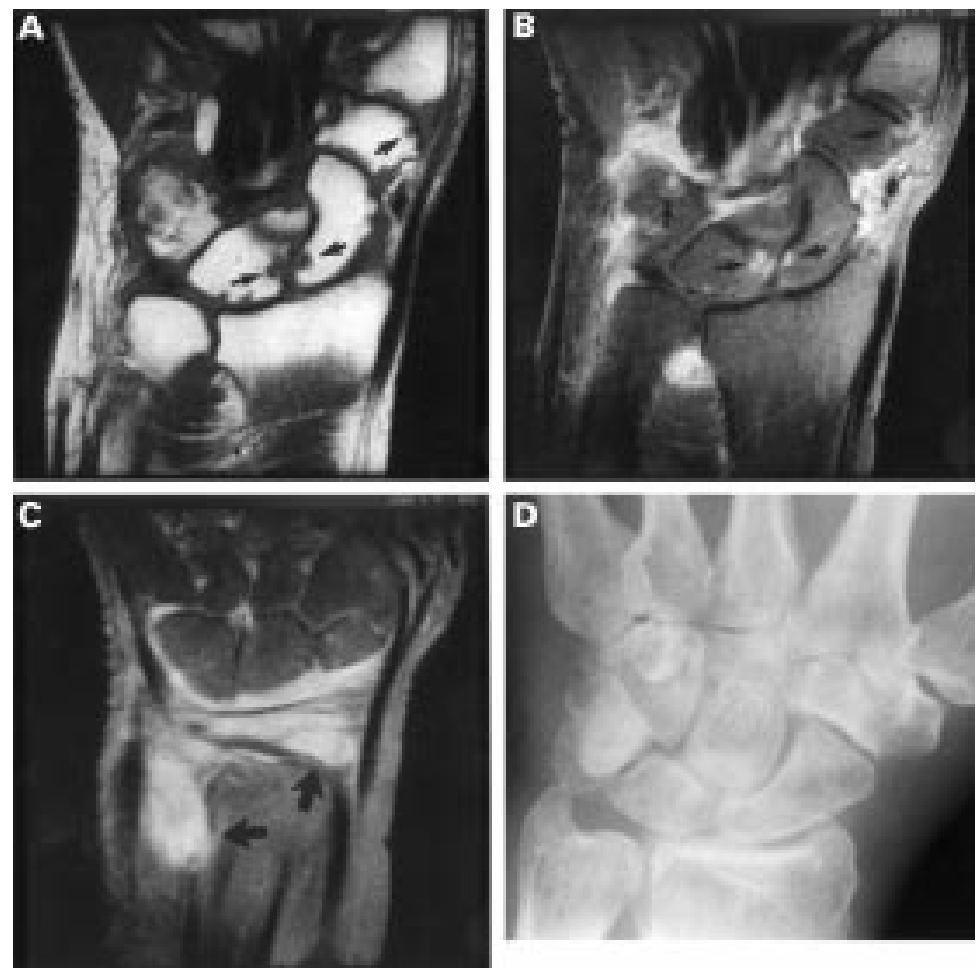

Figure 2 (A) MRI scan of the right wrist of one of the patients. T1 weighted coronal sequence showing erosions in the lunate, scaphoid, and trapezium (black arrows). (B) T1 weighted fat suppressed, post-gadolinium, coronal sequence. Erosions containing enhancing synovium are present in the lunate, scaphoid, trapezium, and triquetrum (black arrows). (C) T1 weighted post-gadolinium coronal sequence. Synovial hypertrophy and enhancement in the distal radioulnar and radiocarpal joints (wide arrows). (D) Plain radiograph of right wrist. No profiled cortical erosions but focal lucencies present in the capitate and scaphoid.

Table 5 MRI erosions compared with radiographic erosions and shared epitope

\begin{tabular}{llll}
\hline & \multicolumn{2}{l}{ MRI of dominant wrist } \\
\cline { 2 - 4 } & $\begin{array}{l}\text { Erosions }(\%) \\
(n=19)\end{array}$ & $\begin{array}{l}\text { No erosions (\%) } \\
(n=23)\end{array}$ & $\begin{array}{l}\text { Total (\%) } \\
(n=42)\end{array}$ \\
\hline $\begin{array}{l}\text { Radiograph of dominant hand } \\
\text { Erosions }\end{array}$ & $5(26.3)$ & $1(4.3)$ & $6(14.3)$ \\
$\begin{array}{l}\text { No erosions } \\
\text { Genotype }\end{array}$ & $14(73.7)$ & $21(91.3)$ & $35(83.3)$ \\
HLADRB ${ }^{*} 04 / 01(\mathrm{SE}+)$ & $16(84.2)$ & $16(69.6)$ & $32(76.2)$ \\
\hline
\end{tabular}

$\star_{n}=41$ (see text).

MRI erosions bearing the shared epitope and those without.

\section{Results}

VALIDATION OF MRI SCORING SYSTEM

Two radiologists scored the 42 baseline MRI wrist scans separately, blinded to results of plain radiographs performed concurrently (table 3). Interobserver reliability for the total MRI score was 0.81 (95\%CI: 0.64, 0.89) with slightly more variation in readings for erosion number; $r=0.79$ (0.64, 0.98), tendonitis; $r=$ $0.77(0.60,0.87)$, and synovitis; $r=0.74(0.56$, $0.85)$. Seven scans were scored again separately by each radiologist, two months later, to give a measure of intraobserver reliability. This was high for both observers (observer 1: total score $r=0.94(0.66,0.99)$, erosion score $r=0.98$ (0.93, 0.99), observer 2: total score $r=0.81$ $(0.05,0.95)$, erosion score $r=0.92(0.48$, $0.98)$.

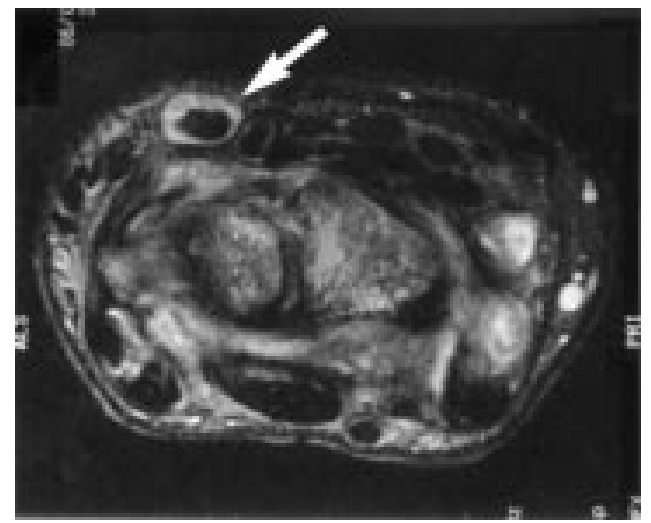

Figure 3 MRI T2 weighted fat suppressed sequence, axial image, to show tendonitis of flexor carpi radialis, which appears as increased signal and thickening of tendon sheath (white arrow).

EROSIONS ON MRI SCANS AND PLAIN RADIOGRAPHS

Erosions were scored as present in 15 of 42 MRI scans (36\%) by observer 1 and 20 of 42 scans $(48 \%)$ by observer 2 . This difference was not statistically significant $(\mathrm{p}=0.3)$. Figure 1 shows the erosion score and total score for each observer. Because of the importance of defining the erosive group as accurately as possible, both observers reviewed scans together for a consensus opinion where they differed as to the presence or absence of erosions. Eleven scans were reviewed, of which seven were agreed to reveal erosions, giving a total of 19 erosive scans or $45 \%$ of the total group. The most frequent site for erosions for both observers was the capitate, which was involved in $67 \%$ and $65 \%$ of erosive scans respectively (identified by one or both observers in $39 \%$ of all scans) (table 4).

Radiographs of the dominant carpus were also read for the presence of erosions by both radiologists, blinded to MRI findings, according to the method described by Sharp. ${ }^{12}$ Only 41 sets of radiographs were available for scoring (one set had been lost). Initial readings were performed separately by each radiologist and in 37 of 41 sets of radiographs, agreement was reached on the presence/absence of erosions (90\% agreement). Those films where radiologists differed were re-read by both observers to obtain a consensus opinion. Nine sets of radiographs were re-evaluated by each radiologist to determine intraobserver scoring consistency. Percentage agreement for observer 1 was $96 \%$ and for observer $2,98.5 \%$.

Radiographic erosions were present in wrist films from six patients (14.6\%) and absent in 35. Table 5 summarises the results compared with findings on MRI scans. In 14 patients, erosions were apparent on MRI scans but not on plain radiographs.

BONE MARROW OEDEMA, SYNOVITIS, TENDONITIS Bone marrow oedema was present according to one or both observers in 27 patients $(64 \%)$. It was most commonly found within the lunate where it was recorded in 14 patients (33\%). Synovitis was observed in 39 scans (93\%); the median score being 6.5 for observer 1 and 9.0 
Table 6 Correlations between MRI scores and clinical indicators of inflammation

\begin{tabular}{llllll}
\hline \multicolumn{5}{c}{ MRI scores } \\
\cline { 2 - 6 } & Erosions & $\begin{array}{l}\text { Bone } \\
\text { oedema }\end{array}$ & Synovitis & Tendonitis & Total \\
\hline Clinical scores & & & & & \\
CRP & $0.35^{\star}$ & $0.35^{\star}$ & $0.42^{\star \star}$ & 0.02 & $0.40^{\star \star}$ \\
ESR & 0.29 & $0.32^{\star}$ & 0.24 & 0.12 & $0.30^{\star}$ \\
Pain score & 0.31 & $0.42^{\star \star}$ & 0.29 & 0.11 & $0.39^{\star}$ \\
Ritchie & $0.32^{\star}$ & $0.40^{\star \star}$ & 0.01 & 0.04 & 0.28 \\
Swollen joint score & 0.22 & 0.21 & 0.05 & 0.24 & 0.19 \\
DAS & $0.32^{\star}$ & $0.38^{\star}$ & 0.18 & 0.17 & $0.33^{\star}$ \\
\hline${ }^{\star} \mathrm{p}<0.05,{ }^{\star \star} \mathrm{p}<0.01$. & & & & &
\end{tabular}

for observer 2. The ulnar aspect of the radiocarpal joint and the pisotriquetral joint were most frequently involved with synovitis $(88 \%)$. Tendonitis was determined by increased signal of the tendon or tendon sheath on $\mathrm{T} 1$ and $\mathrm{T} 2$ images and/or a change in tendon size, and was recorded in 33 scans $(79 \%)$. The most common sites for tendon involvement were extensor carpi ulnaris and flexor carpi radialis (fig 3). Table 4 gives a summary of these results.

MRI EROSION SCORE CORRELATES BEST WITH

BONE MARROW OEDEMA AND CRP

MRI erosion score was highly correlated with other components of the MRI score including bone marrow oedema and synovitis $(r=0.83$ and 0.50 respectively, $\mathrm{p}<0.01$ for both). A weaker correlation was apparent between MRI synovitis and tendonitis scores $(r=0.32$, $\mathrm{p}<0.05)$. MRI scores were also compared with clinical scores (table 6). CRP was the clinical measure most strongly associated with MRI scores (correlated significantly with MRI synovitis score, $r=0.42, \mathrm{p}<0.01$, and total MRI score, $r=0.40, \mathrm{p}<0.01)$. The MRI erosion score correlated with the Ritchie score, disease activity score, and CRP ( $r=0.32,0.32,0.35$ respectively, $\mathrm{p}<0.05$ for all).

MRI EROSIONS COMPARED WITH PRESENCE OF SHARED EPITOPE

HLA-DRB $1{ }^{\star} 04$ or ${ }^{\star} 01$ alleles bearing the shared epitope were present in 32 of 42 patients $(76.2 \%)$ as determined at DNA sequence level. Of those with MRI erosions, 16 of $19(84.2 \%)$ were shared epitope positive compared with 16 of 23 patients $(69.6 \%)$ without MRI erosions (NS, p = 0.3). Gene dose for the shared epitope $(0,1$ or 2 copies) was also not significantly correlated with the presence of MRI erosions.

\section{Discussion}

The gold standard for joint damage in RA is currently the presence of radiological erosions. Management decisions including when to institute aggressive treatment with drugs such as methotrexate and combination regimens are influenced by the presence or absence of erosive change. However, most patients at diagnosis do not have erosions on plain radiography. ${ }^{125}$ There is often a delay of 6 to 12 months before erosions can be confirmed by radiography and during this time the clinician must treat empirically according to clinical indicators of synovitis, which may not accu- rately predict prognosis. ${ }^{46} \mathrm{MRI}$ can reveal erosions as well as synovitis and tendonitis early in RA, giving a detailed picture of joint inflammation and damage..$^{11}{ }^{1122}$ This study of 42 RA patients with recent onset disease (median symptom duration of four months) provides baseline information on MRI changes in the dominant wrist. This is the largest reported study of MRI at this early stage of RA.

We have developed a scoring system for quantifying MRI changes at the wrist in early RA. Rominger et al first proposed such a system in $1993^{27}$ and Ostergaard et al attempted a more detailed assessment of synovitis. $^{22}$ Our system incorporates these features but also quantifies bone marrow oedema, which is specific to MRI scans ${ }^{28}$ and reflects an increase in the water content of the bone marrow. We have expanded the assessment of tendonitis from scoring of the tendon sheath to include the tendon itself, which may swell in response to inflammation leading ultimately to attenuation and rupture. ${ }^{29}$ Like other investigators, we have not attempted to score cartilage thinning as this could not be adequately measured within the small joints of the wrist.

We have examined our scoring system for reliability using two musculoskeletal radiologists, with MRI expertise, who read scans independently. The " $r$ " value of 0.81 for total score interobserver reliability compares favourably with plain radiographic scoring for erosions and joint space narrowing using the Sharp system. ${ }^{38}$ Reliability for erosion number and bone marrow oedema was also high with $r$ values of 0.79 and 0.84 respectively. Scoring for synovitis was less consistent between the two observers $(r=0.74)$ and this reflects difficulty in assessing small changes in synovial thickness and enhancement between the carpal bones. We believe, however, that we have shown sufficient consistency between radiologists for this scoring system to be a useful tool in the interpretation of wrist MRI in early RA.

One of the most important findings from this study was that MRI revealed carpal erosions in $45 \%$ of our group of RA patients at four months from the onset of symptoms while only $15 \%$ had erosions on plain radiographs. This suggests that a much higher percentage of RA patients develop erosions at an earlier stage of disease than has previously been reported radiographically. ${ }^{1}$ Other investigators have also shown MRI to be more sensitive than plain radiographs in the detection of early erosive change in RA. ${ }^{11}{ }^{11}{ }^{30}$ A recent report revealed 18 of 19 patients with recent onset disease (symptoms for less than one year) to have erosions on MRI of the dominant hand. ${ }^{31}$ One concern, however, is that this increased sensitivity may be at the price of reduced specificity and that some of these lesions may not progress to true erosions as seen on plain radiographs. Intraosseous cysts occur in controls and in degenerative joint disease ${ }^{32}$ but can usually be distinguished from erosions as the latter often contain enhancing synovium identified on post-gadolinium T1 weighted sequences. Partial volume artefacts could also be responsible 
for false positives in scoring erosions. To avoid this, care was taken to ensure erosions were present on two different sequences (including axial and coronal views) or more than one adjacent slice on the same sequence. Assessment of erosive change was difficult, however, when there was generalised synovial enhancement adjacent to carpal bones with irregular margins and inevitably some false positives will have been scored. Longitudinal follow up of these patients with repeat MRI scans and radiographs at one year should allow identification of false positives and estimation of the specificity of this technique.

If the MRI erosions we have observed are true erosions, it could be argued that patients with more severe disease are represented disproportionately in our group. This is possible as most were recruited from secondary and tertiary referral centres. The use of the 1987 ARA revised criteria for diagnosis of $\mathrm{RA}^{14}$ may also have caused some bias towards severity as suggested by the high joint swelling scores and percentage of seropositive patients. These criteria, while used universally in establishing a diagnosis of RA, are not ideal for very early disease as they include the presence of nodules and radiographic erosive change, which may only develop after months to years. Our results should therefore be interpreted as applying best to patients in whom a firm diagnosis of RA can be established within six months of symptom onset. While salazopyrin, methotrexate, and prednisone have been shown in some studies to retard the progression of erosions, they are unlikely to have influenced findings in our patients as the majority had started these drugs within the month before enrolment.

Scores from MRI were analysed to determine which sites in the carpus were involved most frequently. The capitate was the most common site for bony erosion, consistent with observations by other investigators. ${ }^{30}$ Likewise, the most common site for synovitis (the ulnar aspect of the radiocarpal joint) has been identified by other groups. ${ }^{22}$ Tendonitis involved extensor carpi ulnaris and flexor carpi radialis in one third of our patients but in none was tendon rupture identified, reflecting the early stage of disease. Correlations were found between components of the MRI score, the most significant being between erosions and bone marrow oedema $(r=0.83)$. Unlike Jevtic et $a l,{ }^{33}$ we did not find this to be rare in RA, but rather it was a frequent appearance, often related to the presence of erosions in carpal bones and adjacent synovial inflammation. Thus bone marrow oedema could represent the intraosseous reaction to erosion of the cortex by synovium and might sometimes precede the development of erosions. MRI erosions and the total MRI score also correlated with clinical and laboratory markers of disease activity including the CRP, suggesting that those with the greatest degree of joint inflammation may be those with the most aggressive disease. ${ }^{2634}$ There has been recent interest in the time integrated CRP as an early predictor for erosions in $\mathrm{RA}^{35}$ and our findings suggest that MRI scores might also be useful in predicting prognosis. Clearly, data from the one year follow up MRI in our patients will be required before this can be determined.

The shared epitope was found in $76 \%$ of our group in keeping with other populations of RA patients. ${ }^{34}{ }^{36}$ This compares with control data from a previous study from our institution that revealed shared epitope positive HLA$\mathrm{DRB}^{\star} 01 /{ }^{\star} 04$ alleles in $40 \%$ of white subjects and $26 \%$ of Polynesians within a New Zealand population. ${ }^{36}$ We found no significant difference in carriage of the shared epitope between those with $\mathrm{MR}$ erosions $(82.4 \%)$ and those without $(69.6 \%)$. This may be a reflection of the small number of patients in this study but others have also found the shared epitope to be only weakly predictive of erosive disease. ${ }^{37} 38$

Cost is a major deterrent when clinicians consider the use of MRI in early RA. Static MRI of the dominant wrist can be obtained for approximately three times the cost of plain radiology of the hands and feet. However, the latter investigation is almost routinely requested at first presentation and can only infrequently identify erosive change. Therefore the extra cost of MRI might be justified if treatment could then be tailored during the first few months of disease to improve long term patient outcome. Certainly, MRI is now accepted as an important imaging modality for similar reasons in, for example, the investigation of knee pain. Our results suggest that expensive genotyping for HLADRB $1^{\star} 04$ alleles may be much less helpful in identifying "high risk" patients and yet this has been proposed as an important investigation in early RA by a number of authors. ${ }^{39}$

In summary, MRI of the wrist in patients with early RA frequently reveals abnormalities implying the presence of erosions, bone marrow oedema, synovitis, and tendonitis. We have found a high prevalence of erosions, even at a very early stage of disease. However, the sensitivity and specificity of this technique for defining erosions with reference to the radiological gold standard await results from the longitudinal study of this group. We have developed a reliable scoring system for quantifying MRI abnormalities and have demonstrated correlations between clinical measures of disease activity and components of the MRI score. This technique provides a means to directly visualise soft tissue inflammation and bony damage in early RA and may be useful in identification of patients requiring aggressive treatment.

The authors wish to acknowledge the assistance of the following clinicians who have referred patients for this study: Dr Mike Butler, Dr David Caughey, Dr Nora Lynch, Dr Alan Doube, Dr Butler, Dr David Caughey, Dr Nora Lynch, Dr Alan Doube, Dr
Hamish Hart, Dr Peter Gow, Dr Raoul Stuart, Dr Terry Macedo, Dr Max Robertson, Dr Roger Reynolds, Dr Bob Grigor. We are also most grateful to the technical staff at the Grigor. We are also most grateful to the technical staff at the
Auckland Radiology Group who performed the MRI and to Ms Auckland Radiology Group who performed the MRI and to Ms
Ma Wei who performed HLA-DRBI ${ }^{\star} 04 /{ }^{\star} 01$ genotyping. This study was supported by grants from the Health Research Council of New Zealand, the Arthritis Foundation of New Zealand, the Auckland Medical Research Foundation, the Auckland Radiology Group and Sanofi-Winthrop.

1 Fex E, Jonsson K, Johnson U, Eberhardt K. Development of radiographic damage during the first $5-6$ years of rheumaradiographic damage during the first 5-6 years of rheuma-
toid arthritis. A prospective follow-up study of a Swedish toid arthritis. A prospective follow-up study

2 Pincus T. Longterm outcomes in rheumatoid arthritis. Br J Rheumatol 1995;34 (suppl 2):59-73. 
3 van der Heijde DM, van Leeuwen MA, van Riel PL, Koster AM, van't Hof MA, van Rijswijk MH, et al. Biannual radiographic assessments of hands and feet in a three year prospective follow up of patients with early rheumatoid arthritis. Arthritis Rheum 1992;35:26-34.

4 van Zeben D, Breedveld FC. Prognostic factors in rheumatoid arthritis. J Rheumatol 1996;23 (suppl 44):31-3.

5 Gregerson PK, Silver J, Winchester RJ. The shared epitope hypothesis: an approach to understanding the molecular genetics of susceptibility to rheumatoid arthritis. Arthritis Rheum 1987;30:1205-13.

6 Weyand CM, Hicok KC, Conn DL, Goronzy JJ. The influence of HLA-DRB 1 genes on disease severity in rheumatoid arthritis. Ann Intern Med 1992;117:801-6.

7 Pincus T, Callahan LF. Formal education as a marker for increased mortality and morbidity in rheumatoid arthritis J Chronic Dis 1985;38:973-84.

8 Lopez-mendez A, Daniel WW, Reading JC, Ward JR, Alarcon GS. Radiographic assessment of disease progression in rheumatoid arthritis patients enrolled in the cooperative systematic studies of the rheumatic diseases program randomized clinical trial of methotrexate, auranofin or a combination of the two. Arthritis Rheum ranofin or a co

9 Rau R, Herborn G, Karger T, Werdier D. Retardation of radiologic progression in rheumatoid arthritis with methotrexate. A controlled study. Arthritis Rheum 1991;34: 1236-44

10 Gilkeson G, Polisson R, Sinclair H, Vogler J, Rice J, Caldwell $\mathrm{D}$, et al. Early detection of carpal erosions in patients with rheumatoid arthritis: a pilot study of magnetic resonance imaging. J Rheumatol 1988;15:1361-6.

11 Foley-Nolan D, Stack JP, Ryan M, Redmond U, Barry C, Ennis $\mathrm{J}$, et al. Magnetic resonance imaging in the Ennis J, et al. Magnetic resonance imaging in the plain film radiographs. Br J Rheumatol 1991;30:101-6.

12 Sharp JT, Young DY, Bluhm GB, Brook A, Brower AC Corbett $M$, et al. How many joints in the hands and wrists should be included in a score of radiological abnormalities used to assess rheumatoid arthritis? Arthritis Rheum 1985; 28:1326-35.

13 Larsen A, Dale K, Eek M. Radiographic evaluation of rheumatoid arthritis and related conditions by standard reference films. Acta Radiol 1977;18:481-91.

14 Arnett FC, Edworthy SM, Bloch DA, McShane DJ, Fries JF, Cooper NS, et al. The ARA 1987 revised criteria for the classification of rheumatoid arthritis. Arthritis Rheum 1988;31:315-24.

15 Ritchie DM, Boyle JA, McInnes JM, Jasani MK, Dalakos TG, Grieveson P, et al. Clinical studies with an articular index for the assessment of joint tenderness in patients with rheumatoid arthritis. Q J Med 1968;37:393-406.

16 Pincus T, Summey JA, Soraci SA, Hummon NP, Wallston KA. Assessment of patient satisfaction in activities of daily living using a modified Stanford Health Assessment Questionnaire. Arthritis Rheum 1983;26:1346-53.

17 Steinbrocker O, Traeger CH, Batterman RC. Therapeutic criteria in rheumatoid arthritis. JAMA 1949;140: 659-62.

18 van der Heidje DM, van't Hof MA, van Riel PL, Theunisse LA, Lubberts EW, van Leeuwen MA, et al. Judging disease activity in clinical practice in rheumatoid arthritis: first step activity in clinical practice in rheumatoid arthritis: first step in the development of

19 Olerup O, Zetterquist H. HLA-DR typing by PCR amplification with sequence specific primers (PCR-SSP) in 2 hours: An alternative to serological DR typing in clinical practice including donor-recipient matching in cadaveric transplantation. Tissue Antigens 1992;39:225-35.

20 Voorter CEM, Rozemuller EH, de Bruyn-Geraets D, van der Zwan A-W, Tilanus MGJ, van den Berg-Loonen EM. Comparison of DRB sequence-based typing using different strategies. Tissue Antigens 1997;49:471-6.
21 Gao X, Olsen NJ, Pincus T, Stastny P. HLA-DR alleles with naturally occurring amino acid substitutions and risk for development of

22 Ostergaard M, Gideon P, Sorensen K, Hansen M, Stoltenberg $\mathrm{M}$, Henricksen $\mathrm{O}$, et al. Scoring of synovial membrane hypertrophy and bone erosions by MR imaging in clinically active and inactive rheumatoid arthritis of the wrist. Scand J Rheumatol 1995;24:212-18.

23 Shrout PE, Fleiss JL. Intraclass correlations: Uses in assessing rater reliability. Psychol Bull 1979;86:420-8.

24 Snedecor G, Cochrane W. Statistical methods. 6th ed. Iowa: Iowa State University Press, 1967.

25 Gough A, Faint J, Salmon M, Hassell A, Wordsworth P, Dilling D, et al. Genetic typing of patients with inflammatory arthritis at presentation can be used to predict outcome. Arthritis Rheum 1994;37:1166-70.

26 McQueen FM, Tan PLJ. Predicting disease severity in rheumatoid arthritis. NZ Med J 1994;107:124-6.

27 Rominger MB, Bernreuter WK, Kenney PJ, Morgan SL, Rominger MB, Bernreuter WK, Kenney PJ, Morgan SL,
Blackburn WD, Alarcon GS. MR Imaging in early Blackburn WD, Alarcon GS. MR Imaging in early
rheumatoid arthritis: preliminary results. Radiographics 1993;13:37-46.

28 Jevtic V, Watt I, Rozman B, Kos-Golja M, Demsar F, Jarh O. Distinctive radiological features of small hand joints in rheumatoid arthritis and seronegative spondyloarthritis demonstrated by contrast-enhanced (Gd-DPTA) magnetic resonance imaging. Skeletal Radiol 1995;24:351-5.

29 Rubens DJ, Blebea JS, Totterman SM, Hooper MM. Rheumatoid arthritis: evaluation of wrist extensor tendons with clinical examination versus MR imaging - a preliminary report. Radiology 1993;187:831-8.

30 Jorgensen C, Cyteval C, Anaya JM, Baron MP, Lamarque JL, Sany J. Sensitivity of magnetic resonance imaging of the wrist in very early rheumatoid arthritis. Clin Exp Rheumatol 1993;11:163-8.

31 McGonagle D, Green MJ, Proudman S, Richardson C, Veale D, O'Connor P, et al. The majority of patients with rheumatoid arthritis have erosive disease at presentation when magnetic resonance imaging of the dominant hand is employed. Br J Rheumatol 1997;36 (suppl 1):121.

32 Tonolli-Serabian I, Poet JL, Dufour M, Carasset S, Mattei JP, Roux H. Magnetic imaging of the wrist in rheumatoid arthritis: comparison with other inflammatory joint diseases and control subjects. Clin Rheumatol 1996;15:13742

33 Jevtic V, Rozman B, Watt I, Presetnik M. Use of contrast enhanced MRI in the assessment of therapeutic response to a disease-modifying antirheumatic drug. Br J Rheumatol 1995;34:956-9.

34 van der Heidje DM, van Riel PL, van Rijswijk MH, van de Putte LB. Influence of prognostic factors on the final outcome in rheumatoid arthritis: a review of the literature. Semin Arthritis Rheum 1988;17:284-92.

35 van Leeuwen MA, van Rijswijk MH, Sluiter WJ, Piet LC, van Riel PL, Kuper $\mathrm{IH}$, et al. Individual relationship between progression of radiological damage and the acute phase response in early rheumatoid arthritis. Towards development of a decision support system. J Rheumatol development of

36 Tan PLJ, Farmiloe S, Roberts M, Guersen A, Skinner MA. HLA-DR4 subtypes in New Zealand Polynesians. Predominance of DW13 in the healthy population and association of DW15 with rheumatoid arthritis. Arthritis Rheum 1993;36:15-19.

37 Eberhardt K, Fex E, Wollheim FA. Associations of HLA-DRB and DQB genes with 2 and 5 year outcome in rheumatoid arthritis. Ann Rheum Dis 1996;55:34-9.

38 Higami K, Hakoda M, Matsuda Y, Ueda H, Kashiwazaki S. Lack of association of HLA-DRB1 genotype with radiologic progression in Japanese patients with early rheumatoid arthritis. Arthritis Rheum 1997;40:2241-7.

39 Nepom GT, Gersuk V, Nepom BS. Prognostic implications of HLA genotyping in the early assessment of patients with rheumatoid arthritis. J Rheumatol 1996;23 (suppl 44):5-9. 